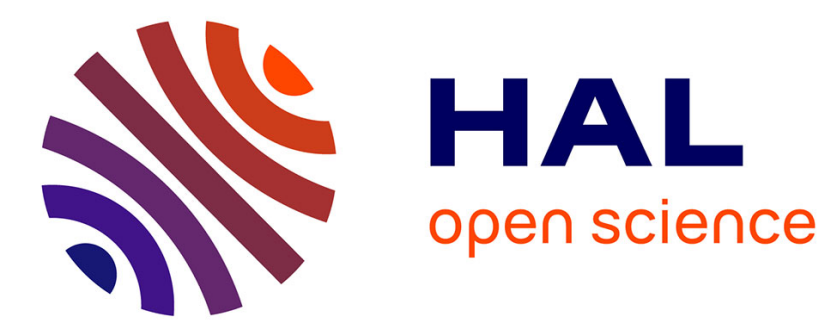

\title{
L'intégration logistique au sein d'une chaîne multi-acteurs : le cas de Renault Trucks
}

Christopher Melin

\section{To cite this version:}

Christopher Melin. L'intégration logistique au sein d'une chaîne multi-acteurs: le cas de Renault Trucks. 3ème conférence annuelle d'Atlas/AFMI Association Francophone de Management International " ANCRAGES CULTURELS ET DYNAMIQUES DU MANAGEMENT INTERNATIONAL

", Jul 2013, Montréal, Canada. halshs-00879437

\section{HAL Id: halshs-00879437 https://shs.hal.science/halshs-00879437}

Submitted on 3 Nov 2013

HAL is a multi-disciplinary open access archive for the deposit and dissemination of scientific research documents, whether they are published or not. The documents may come from teaching and research institutions in France or abroad, or from public or private research centers.
L'archive ouverte pluridisciplinaire HAL, est destinée au dépôt et à la diffusion de documents scientifiques de niveau recherche, publiés ou non, émanant des établissements d'enseignement et de recherche français ou étrangers, des laboratoires publics ou privés. 


\section{L'INTEGRATION LOGISTIQUE AU SEIN D'UNE CHAINE MULTI-ACTEURS : LE CAS DE RENAULT TRUCKS}

\section{Christopher MELIN}

ATER en stratégie

IAE Lyon, Université Jean Moulin Lyon 3

6 cours Albert Thomas, 69008 Lyon

christopher.melin@univ-lyon3.fr

\section{Résumé}

Cet article vise à présenter les résultats d'une recherche-action conduite au sein de Renault trucks, filiale du groupe Volvo sur la notion d'intégration logistique au sein d'une chaîne multi-acteurs. A l'aide d'une grille de lecture multi-niveaux et d'une analyse dysfonctionnelle issue de 29 entretiens, il est montré les principaux défis qu'une firme multinationale du secteur des poids lourds doit relever dans le pilotage d'une chaîne logistique multi-acteurs. D'autre part, l'élaboration d'un outil de diagnostic et d'aide à l'action stratégique nous permet de mettre en avant la variable autonomie/hétéronomie du partenaire dans la volonté d'une intégration logistique.

\section{Mots clés :}

Intégration Logistique ; Chaîne Logistique Multi-acteurs ; Relations Intra- et Interorganisationnelles ; Renault Trucks 


\section{INTRODUCTION}

Dans un contexte de globalisation et de reconfiguration des zones géographiques de l'économie mondiale, de nombreuses FMN sont amenées à rechercher des avantages concurrentiels à tous les niveaux de la chaîne de valeur (Bartlett et Beamish, 2011) en révisant, entre autres, la localisation de leurs activités internationales (Mayrhofer et Urban, 2011). L'objectif des FMN semble se tourner sur la conception de systèmes de production souples et flexibles (Buckley et Ghauri, 2004) à l'échelle mondiale, afin de répondre aux besoins de nouveaux consommateurs qui ne sont plus seulement localisés sur les pays d'origine de celles-ci. La fonction de production est alors souvent externalisée auprès de partenaires internationaux qui assemblent le produit à proximité des clients finaux. Ce système permet de réduire les coûts fixes et d'adapter les produits aux exigences des marchés locaux (Mayrhofer, 2011). Au niveau mondial, ce phénomène provoque un éclatement de la chaîne de valeur (Porter, 1985) dont les activités complémentaires dans la conception, la production et la commercialisation d'un produit donné sont dispersées géographiquement.

Le management logistique s'est ainsi inséré progressivement dans des organisations de plus en plus complexes avec une gestion d'interfaces non plus uniquement intra-organisationnelle mais intra- et inter-organisationnelles (Colin, 2005). La coordination de la chaîne logistique est devenue stratégiquement importante en supply chain management (Fabbe-Costes et Lancini, 2009). De plus, la coordination et l'intégration des activités internationales correspondent aux principaux défis des entreprises du secteur automobile (Gereffi, et al., 2005). Face à ces constats, cet article a pour objectif d'étudier la notion d'intégration logistique au sein d'une chaîne multi-acteurs à un double niveau, intra- et interorganisationnel. En nous appuyant sur une recherche-action conduite au sein de Renault Trucks, filiale du groupe Volvo, l'un des leaders mondiaux sur son secteur d'activité, nous 
cherchons à comprendre comment une FMN peut-elle intégrer la fonction logistique au sein d'une même chaîne multi-acteurs et ce, à un double niveau : intra- et inter-organisationnels.

Pour ce faire, nous commençons par poser les bases d'une grille d'analyse multi-niveaux de la notion d'intégration logistique afin de révéler dans un second temps les principaux défis dans le pilotage d'une chaîne logistique multi-acteurs. Puis, nous présentons la méthodologie de recherche-action conduite avec Renault Trucks. Dans un troisième temps, nous décrivons les principaux résultats de cette recherche pour terminer par une discussion autour de ces résultats.

\section{De la Chaîne Globale de Valeur au Supply Chain Management}

La chaîne de valeur des FMN implique aujourd'hui une multitude d'acteurs éparpillés à travers le monde, faisant évoluer leur analyse d'un niveau intra-organisationnel à un niveau inter-organisationnel. Ces chaînes de valeur transnationales sont ainsi enchevêtrées dans des réseaux intra- et inter-entreprises de plus en plus complexes. L'approche de la chaîne globale de valeur $(\mathrm{CGV})^{1}$ se veut un outil d'analyse de la structure de gouvernance qui décrit le processus par lequel certains acteurs de la chaîne exercent un contrôle sur d'autres participants et la manière dont ces «firmes pilotes » s'approprient ou distribuent la valeur créée le long de la chaîne (Bair, 2010). Cette approche semble ainsi être une grille de lecture adaptée pour l'analyse des liens particuliers au sein de la chaîne notamment en termes de mécanismes de coordination inter-firmes (Sturgeon, 2009). En effet, l'approche CGV permet de décrire la séquence des processus par lesquels les biens et produits sont conçus, fabriqués et mis sur le marché (Bair, 2010) à un niveau global intégrant tous les acteurs impliqués dans ces processus, aussi bien à un niveau intra-organisationnel, qu'inter-organisationnel.

\footnotetext{
${ }^{1}$ Issue des travaux du sociologue Gereffi (1994), initialement appelée chaîne globale de commodité (CGC) puis repris et développé par le terme de chaîne globale de valeur (CGV), (Gereffi, et al. 2005 ; Sturgeon, 2009).
} 
Par ailleurs, au-delà du phénomène de mondialisation et de reconfiguration des localisations des activités de la chaîne de valeur (Colovic et Mayrhofer, 2011), les organisations s'intéressent plus particulièrement à la fonction logistique de la CGV en termes de gestion du flux global des produits, des marchandises et des services, ainsi que des échanges qui en découlent (Brulhart et Favoreu, 2006). L'attention est donc portée sur la chaîne (ou supply chain) qui «correspond à l'ensemble des flux physiques de produits (et des flux d'information associés) qui structurent les relations d'échange entre entreprises, du fournisseur $d u$ fournisseur jusqu'au client du client. Y sont intégrées des activités telles que le transport, l'entreposage et le stockage, la manutention, la préparation des commandes, etc. » (Fulconis, et al., 2011 : 16). D’une vision fragmentée de la chaîne logistique à une approche transversale inter-fonctions de la gestion des flux logistique des organisations (niveau intraorganisationnel) observée dans les années 1960-1980, le management de la chaîne logistique s'est peu à peu tourné sur une visions transversale de gestion par les flux à un double niveau, intra- et inter-organisationnel dans les années 1990 à 2000 (Colin, 2005). Ainsi, cette prise de conscience que les flux logistiques ne s'arrêtaient pas aux frontières de l'entreprise, a conduit les chercheurs et professionnels à se positionner sur le management d'une chaîne logistique intra- et inter-organisationnelle (Livolsi, 2009), aussi appelée : chaîne logistique multi-acteurs (Paché et Spalanzani, 2007). La mise en place d'un management de la logistique à ce double niveau autour d'un objectif commun s'insère dans le concept de supply chain management (SCM) comme le définit le Council of Supply Chain Management Professionals ${ }^{2}$ (CSCMP) : « le supply chain management comprend la planification et la gestion de toutes les activités impliquées dans la recherche de fournisseurs, de l'approvisionnement, de la transformation et toutes les activités relevant du management logistique. (...) Le SCM est une fonction intégratrice qui vise à relier les fonctions et les processus clés de l'organisation à un double

\footnotetext{
${ }^{2}$ http://www.cscmp.org - visité le 29.07.2013
} 
niveau, intra- et inter-organisationnel afin d'élaborer un business model performant et cohérent».

\section{L'intégration intra et inter-organisationnelle des acteurs du SCM}

Comme nous avons pu le voir précédemment, la notion d'intégration est très présente en logistique. A ce titre, selon Fabbe-Costes (2007), pour étudier la fonction intégratrice du SCM, il incombe de répondre à deux questions spécifiques. D'une part, il est important de questionner le «quoi intégrer? » et, d'autre part, de se positionner sur «l'étendue de cette intégration ». Afin de répondre à la question du « quoi intégrer ? », une première analyse peut se faire selon quatre couches (cf. tableau 1): une intégration des flux, des processus et activités, des systèmes et technologies et des acteurs (organisations).

Tableau 1 : SCM : quatre couches d'intégration à considérer (tiré de Fabbe-Costes, 2007 : 27)

\begin{tabular}{|c|c|}
\hline $\begin{array}{c}\text { Quatre couches } \\
\text { d'intégration }\end{array}$ & Caractéristiques de l'intégration pour chaque couche \\
\hline Flux & $\begin{array}{c}\text { Fluidité et continuité, pertinence des flux physiques, } \\
\text { informationnels et financiers, individuellement et de manière } \\
\text { combinée. }\end{array}$ \\
\hline $\begin{array}{c}\text { Processus et activités } \\
\text { Systèmes et } \\
\text { technologies }\end{array}$ & $\begin{array}{c}\text { Synchronisation des opérations pour chaque processus clé; } \\
\text { cohérence entre processus opérationnels clés, processus de pilotage et } \\
\text { processus support; intégration des processus au niveau opérationnel, } \\
\text { organisationnel et stratégique. }\end{array}$ \\
\hline Acteurs (organisations) & $\begin{array}{c}\text { Interopérabilité et interconnectivité des systèmes et technologies } \\
\text { physiques et d'information, individuellement et de manière combinée. }\end{array}$ \\
\hline $\begin{array}{c}\text { Interaction, coordination et collaboration des individus, des } \\
\text { collectif, structures interfaces ou partagées, congruence stratégique, } \\
\text { organisationnelle et culturelle. }\end{array}$ \\
\hline
\end{tabular}

Pour répondre à la question liée à l'étendue de l'intégration au sein du SCM, en s'appuyant sur des travaux d'autres chercheurs, cet auteur propose cinq niveaux d'intégration que nous résumons par le tableau 2. 
Tableau 2: Les cinq niveaux d'intégration des chaînes logistiques (adapté de Fabbe-Costes, 2007)

\begin{tabular}{|c|c|c|c|c|c|}
\cline { 2 - 6 } \multicolumn{1}{c|}{} & \multicolumn{4}{c|}{ Les différents niveaux d'intégration de la chaîne logistique } \\
\cline { 2 - 6 } & $\begin{array}{c}\text { Intra- } \\
\text { organisationnelle }\end{array}$ & $\begin{array}{c}\text { Inter- } \\
\text { organisationnelle } \\
\text { limitée }\end{array}$ & $\begin{array}{c}\text { Inter- } \\
\text { organisationnelle } \\
\text { étendue }\end{array}$ & $\begin{array}{c}\text { Multi- } \\
\text { chaînes }\end{array}$ & Sociétale \\
\hline & $\begin{array}{c}\text { Aux partenaires } \\
\text { directs (les }\end{array}$ & $\begin{array}{c}\text { A l'ensemble des } \\
\text { partenaires d'une } \\
\text { chaîne }\end{array}$ & $\begin{array}{c}\text { A l'ensemble } \\
\text { des chaînes } \\
\text { auxquelles } \\
\text { participe une } \\
\text { organisation }\end{array}$ & $\begin{array}{c}\text { Avec une perspective } \\
\text { de développement } \\
\text { durable et qui } \\
\text { s'intéresse à des } \\
\text { parties prenantes } \\
\text { externes à la châne } \\
\text { logistique }\end{array}$ \\
\hline
\end{tabular}

En croisant les différentes couches et niveaux d'intégration au sein des chaînes logistiques évoqués précédemment, il est alors possible de déterminer un degré d'intégration et des facteurs facilitateurs de l'intégration logistique. Dans le cadre de ce travail de recherche, nous nous intéressons plus spécifiquement à la notion d'intégration entre acteurs/organisations d'une même chaîne logistique plus particulièrement entre une FMN et ses partenaires internationaux pour l'assemblage de produits sur des marchés hors Europe. C'est la raison pour laquelle, nous nous focalisons selon le cadre d'analyse proposé par Fabbe-Costes (2007), sur une intégration intra-organisationnelle (inter-fonctions au sein de la FMN étudiée) et une intégration inter-organisationnelle limitée aux premiers clients (les partenaires internationaux), (cf. tableau 3).

Tableau 3 : Grille d'analyse de l'intégration logistique (adapté de Fabbe-Costes, 2007)

\begin{tabular}{|l|c|l|l|}
\hline \multicolumn{2}{|c|}{ Etendue $\rightarrow$} & \multicolumn{1}{c|}{$\begin{array}{c}\text { Intégration } \\
\text { intra-organisationnelle }\end{array}$} & \multicolumn{1}{c|}{$\begin{array}{c}\text { Intégration } \\
\text { inter-organisationnelle limitée }\end{array}$} \\
\hline $\begin{array}{l}\text { Acteurs } \\
\text { Individus } \\
\text { Equipes }\end{array}$ & $\begin{array}{c}\text { Degré } \\
\text { d'intégration }\end{array}$ & $\begin{array}{l}\text { - Degré d'intégration entre les } \\
\text { individus, les équipes, les fonctions } \\
\text { au sein de chaque entreprise }\end{array}$ & $\begin{array}{l}\text { - Degré d'intégration avec les } \\
\text { partenaires amont et aval directs }\end{array}$ \\
\hline
\end{tabular}




\begin{tabular}{|c|c|c|c|}
\hline \begin{tabular}{|l|} 
Fonctions \\
Entreprises
\end{tabular} & $\begin{array}{c}\text { Facteurs } \\
\text { facilitateurs } \\
\text { d'intégration }\end{array}$ & $\begin{array}{l}\text { - Insertion des individus dans des } \\
\text { réseaux sociaux larges } \\
\text { - Capacité à se représenter les } \\
\text { chaînes, les réseaux } \\
\text { - Capacité à prendre le leadership } \\
\text { de parties de chaîne } \\
\text { - Culture projet, risque, } \\
\text { changement, innovation }\end{array}$ & $\begin{array}{l}\text { - Capacité à mettre en place des } \\
\text { structures inter-organisationnelles } \\
\text { stables ou temporaires } \\
\text { - Choix de partenaires fiables, sûrs } \\
\text { et flexibles } \\
\text { - Entretien des relations avec les } \\
\text { partenaires critiques et/ou } \\
\text { stratégiques }\end{array}$ \\
\hline
\end{tabular}

En pratique, les questions liées à la mesure du degré d'intégration ou la proposition d'outils de gestion qui permettent de rendre effectif un management par les processus ne semblent pas encore trouver de réponses satisfaisantes (Paché et Spalanzani, 2007). De plus, la fonction intégratrice du SCM comme définie par le CSCMP, qui vise à relier les fonctions et les processus clés de l'organisation dans la poursuite d'un objectif commun, pose la question du pilotage des relations intra- et inter-organisationnelles au sein de la chaîne logistique multiacteurs. A ce titre, certains auteurs ont montré que la mise en place de dispositifs de contrôle et d'évaluation s'avère nécessaire et utile pour maintenir des relations partenariales (Brulhart et Favoreu, 2006). Afin de compléter certains travaux sur la question du pilotage des relations intra et inter-organisationnelles au sein du SCM et en nous intéressant aux relations entre les différentes fonctions et sites détenus par une FMN (niveau intra-organisationnel) et ses partenaires internationaux (niveau inter-organisationnel), nous présentons le cas d'une recherche-action conduite au sein de Renault Trucks, filiale du groupe Volvo. Nous proposons ainsi trois niveaux d'analyse pour l'étude du pilotage des relations entre une FMN et ses partenaires internationaux au sein d'une même chaîne logistique : l'approche CGV, l'intégration intra-organisationnelle et l'intégration inter-organisationnelle limitée.

\section{METHODOLOGIE}

Nos propos se basent sur une étude de cas approfondie (Yin, 2003) d'une firme multinationale (FMN) faisant partie des leaders mondiaux dans la fabrication et la commercialisation de 
camions à l'échelle mondiale : Renault Trucks, filiale du groupe Volvo. Notre choix s'est portée sur une FMN issue de l'industrie automobile plus particulièrement du secteur des poids lourds, qui fait partie avec la grande distribution, des secteurs les plus pertinents à étudier pour illustrer les difficultés de pilotage transversal des chaînes logistiques multi-acteurs (Livolsi, 2007). L'étude du cas ici présentée, s'inscrit dans le cadre d'une recherche-action s'étalant sur une période de 11 mois consécutifs à raison de deux à trois jours par semaine de présence physique sur le terrain d'investigation. Notre travail s'inscrit dans une construction concrète de la réalité en cherchant à élaborer des connaissances actionnables par les acteurs de l'entreprise (Argyris, 1995; David, 2008). Le processus de notre recherche-action se décompose en trois phases, que nous présentons dans le tableau suivant, en précisant pour chacune d'elles, les objectifs et choix méthodologiques adoptés (cf. tableau 4).

Tableau 4 : Le processus de recherche au sein de Renault Trucks

\begin{tabular}{|c|c|c|}
\hline $\begin{array}{c}\text { Principales } \\
\text { Etapes }\end{array}$ & Choix réalisés & Objectifs \\
\hline \multirow{2}{*}{$\begin{array}{c}\text { - PHASE } 1 \text { - } \\
\text { Analyse et } \\
\text { modélisation } \\
\text { du problème } \\
\text { complexe }\end{array}$} & $\begin{array}{l}\text { * } 29 \text { entretiens semi-directifs avec les } \\
\text { principaux acteurs impliqués dans la } \\
\text { chaîne logistique de l'activité } \\
\text { internationale de Renault Trucks. }\end{array}$ & $\begin{array}{l}\text { Relever les principaux } \\
\text { dysfonctionnements rencontrés par les } \\
\text { acteurs de l'entreprise dans la } \\
\text { coordination des relations entre les } \\
\text { différentes entités (intra et inter) au sein } \\
\text { de la chaîne logistique de Renault Trucks. }\end{array}$ \\
\hline & $\begin{array}{l}\text { * Organisation d'une journée de travail } \\
\text { avec les principaux acteurs impliqués } \\
\text { dans la chaîne logistique multi-acteurs } \\
\text { de Renault Trucks ( } 25 \text { participants); } \\
\text { * Analyse des dysfonctionnements par } \\
\text { groupe de discussion interactif ( } 5 \\
\text { groupes/3,5 heures) afin de construire } \\
\text { une représentation commune du } \\
\text { problème complexe. }\end{array}$ & $\begin{array}{l}\text { Interprétation des acteurs de l'entreprise } \\
\text { sur les causes racines des } \\
\text { dysfonctionnements sous forme de } \\
\text { groupe de discussion interactif (Balogun, } \\
\text { et al., 2003) afin de collecter une } \\
\text { représentation intelligible du problème } \\
\text { complexe par une intersubjectivité } \\
\text { contradictoire (Savall et Zardet, 1997). }\end{array}$ \\
\hline
\end{tabular}




\begin{tabular}{|c|c|c|}
\hline $\begin{array}{l}\text { - PHASE } 2 \text { - } \\
\text { Elaboration } \\
\text { d'un outil } \\
\text { d'aide au } \\
\text { diagnostic } \\
\text { (janvier 2012 - } \\
\text { avril 2012) }\end{array}$ & $\begin{array}{l}\text { * } 14 \text { entretiens directifs avec les } \\
\text { responsables des différentes fonctions } \\
\text { qui composent le système de } \\
\text { production de Renault trucks; } \\
\text { * Organisation de } 3 \text { réunions de travail } \\
\text { avec des responsables chef de projets } \\
\text { pour effectuer un test de l'outil et le } \\
\text { modifier. }\end{array}$ & $\begin{array}{l}\text { Construction et test d'un outil d'aide au } \\
\text { diagnostic et à la prise de décision } \\
\text { stratégique. Co-construction progressive } \\
\text { du problème et prise de conscience } \\
\text { collective (Chanal, et al., 1997). }\end{array}$ \\
\hline $\begin{array}{l}\text { - PHASE } 3 \text { - } \\
\text { Confrontation } \\
\text { de l'outil et } \\
\text { bouclage } \\
\text { théorique } \\
\text { (mai } 2012 \text { - } \\
\text { juillet 2013) }\end{array}$ & $\begin{array}{l}\text { * Organisation d'une journée de travail } \\
\text { ( } 7 \text { heures) avec les principaux acteurs } \\
\text { de la chaîne logistique ( } 24 \\
\text { participants) ; } \\
* 4 \text { entretiens avec le directeur du } \\
\text { département manufacturing } \\
\text { international et visites de l'entreprise } \\
\text { suite à la recherche-action. }\end{array}$ & $\begin{array}{l}\text { Confronter l'outil à l'avis collectif et mise } \\
\text { en action de l'outil construit sur deux cas } \\
\text { d'assembleurs. Choix et mise en place de } \\
\text { mécanismes de coordination intra- et } \\
\text { inter- organisationnels; } \\
\text { suivi et analyse de l'actionnabilité des } \\
\text { connaissances, validité externe des } \\
\text { résultats et visibilité de la recherche. }\end{array}$ \\
\hline
\end{tabular}

Les 29 entretiens semi-directifs de la PHASE 1 représentent les principaux acteurs impliqués dans la chaîne logistique de l'activité internationale et qui sont en relation avec les partenaires internationaux (cf. tableau 5). Ceci exclu les relations entretenues entre Renault Trucks et ses fournisseurs en amont de la chaîne logistique.

Tableau 5 : Les 29 entretiens semi-directifs

\begin{tabular}{|l|c|}
\hline \multicolumn{1}{|c|}{ Fonctions des interviewés } & $\begin{array}{c}\text { Durée } \\
\text { (en mns) }\end{array}$ \\
\hline Entretiens individuels & 60 \\
\hline Animateur réseau qualité process & 45 \\
\hline Apprenti logistique & 90 \\
\hline Chef de projet marketing à l'international & 90 \\
\hline $\begin{array}{l}\text { Chef de projets industriels à l'international - zone géographique : } \\
\text { Iran, Turquie, Uruguay }\end{array}$ & 90 \\
\hline $\begin{array}{l}\text { Chef de projets industriels à l'international - zone géographique : } \\
\text { Afrique du Sud, Maroc, Tunisie }\end{array}$ & 75 \\
\hline $\begin{array}{l}\text { Chef de projets industriels à l'international - zone géographique : } \\
\text { Soudan, Irak, Malaisie, Taiwan }\end{array}$ & 60 \\
\hline $\begin{array}{l}\text { Chef de projets industriels à l'international - zone géographique : } \\
\text { Russie }\end{array}$ & 30 \\
\hline Controleur de gestion siège & 75 \\
\hline Directeur assurance qualité & 60 \\
\hline Directeur commercial du service international & 90 \\
\hline Directeur international manufacturing & \\
\hline
\end{tabular}




\begin{tabular}{l|c} 
Gestionnaire électronique & 40 \\
\hline Human Resource Business Partner services centraux & 60 \\
\hline Ingénieur industriel pour l'international & 40 \\
\hline $\begin{array}{l}\text { Manager plateforme internationale de Bourg-en-Bresse } \\
\text { (entretien par téléphone) }\end{array}$ & 60 \\
\hline $\begin{array}{l}\text { Manager plateforme internationale de Blainville } \\
\text { (entretien par téléphone) }\end{array}$ & 45 \\
\hline Manager Produit-Process & 140 \\
\hline Manager qualité usines d'assemblages & 60 \\
\hline Responsable Back Office & 30 \\
\hline $\begin{array}{l}\text { Responsable des ingénieurs en outillage pour les usines } \\
\text { d'assemblages internationales }\end{array}$ & 85 \\
\hline Responsable documentation filiales & 45 \\
\hline Responsable juridique partenariats internationaux & 30 \\
\hline Responsable logistique plateforme internationale & 60 \\
\hline $\begin{array}{l}\text { Responsable logistique pour les usines d'assemblages } \\
\text { internationales }\end{array}$ & 60 \\
\hline Responsable Qualité plateforme internationale de Bourg-en-Bresse & 45 \\
\hline Technicien électronique & 45 \\
\hline Technicien service adaptation & 45 \\
\hline Technicien service adaptation & 40 \\
\hline \begin{tabular}{l} 
Entretiens de groupe \\
\hline $\begin{array}{l}\text { Directeur usine d'assemblage Maroc + Front Office Uruguay + } \\
\text { manager fonctionnel (3 personnes interviewées) }\end{array}$
\end{tabular} \\
\hline $\begin{array}{l}\text { Techniciens méthodes / documentation / électroniques et le } \\
\text { responsable documentation usines d'assemblages internationales } \\
\text { (4 personnes interviewées) }\end{array}$ & 60 \\
\hline
\end{tabular}

Nous avons demandé à nos interviewés de se positionner sur les principales difficultés auxquelles ils font face dans le pilotage des relations intra- et inter-organisationnelles au sein de la chaîne logistique notamment avec leurs partenaires internationaux, qui part de la commande jusqu'à la livraison du produit au client final. La durée des entretiens est comprise entre 60 et 140 minutes et ils ont été réalisés entre le mois de septembre et décembre 2011. Une prise de note exhaustive des entretiens a été réalisée et une relecture des entretiens a été effectuée avec une analyse a posteriori limitée à quelques jours. Notre guide d'entretien a été élaboré avec le directeur du département manufacturing international de Renault Trucks et s'est établie autour de la question suivante: quels sont les anomalies/difficultés que vous rencontrez dans le pilotage des relations avec vos partenaires internationaux ? Une distinction 
a été faite entre deux moments particuliers dans le déroulement du processus de fabrication et de commercialisation de véhicules industriels, la vie projet et la vie série. La vie projet correspond au déroulement d'un projet de fabrication et de distribution d'un nouveau produit pour un marché spécifique dont l'assemblage du produit finit se réalise par l'intermédiaire d'un assembleur (partenaire international). La vie série correspond au suivi de l'assemblage d'un produit chez un partenaire international notamment en termes de logistique et qualité produit/process. La vie série et la vie projet constituent les différentes phases rencontrées par les acteurs de la chaîne logistique. Nous avons entamé une réflexion et une analyse conjointe des dysfonctionnements avec les acteurs de l'entreprise lors d'une journée de travail, afin de mettre en avant les principaux défis que Renault Trucks s'efforce de relever dans sa volonté de piloter les relations entretenues avec ses différents partenaires internationaux au sein de sa chaîne logistique multi-acteurs. Ainsi, afin de mettre en avant ces principaux défis, une analyse dysfonctionnelle des 29 entretiens de la PHASE 1 a été conduite (cf. figure 1).

Figure 1 : Analyse dysfonctionnelle de nos 29 entretiens semi-directifs

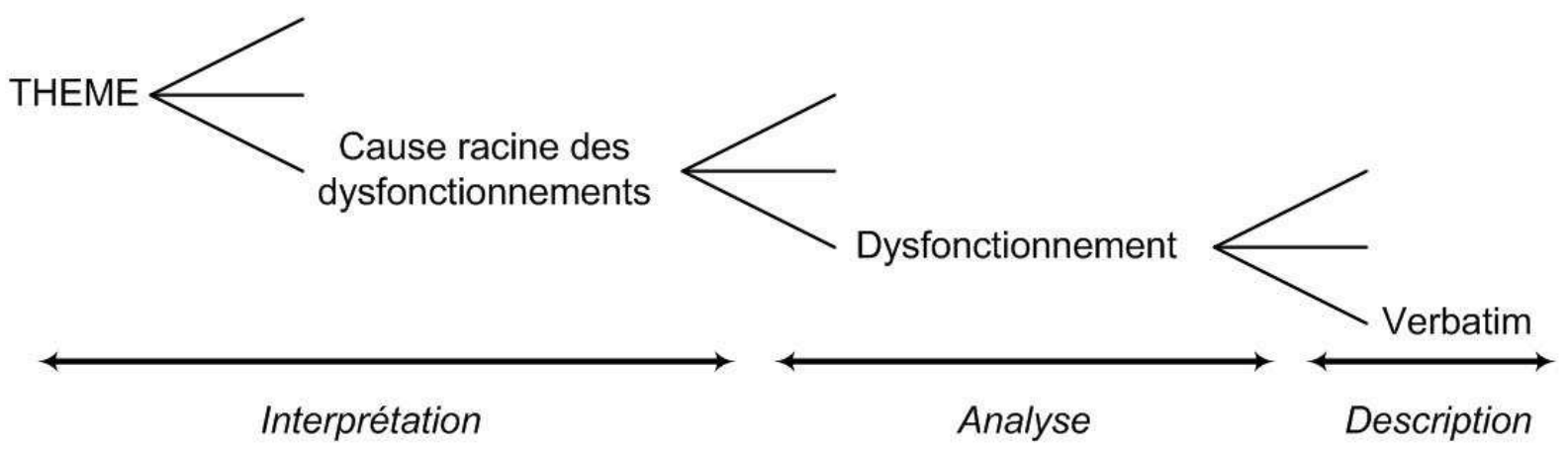

Nous avons ainsi utilisé un codage thématique de nos données en utilisant un raisonnement de type inductif qui consiste à découvrir des régularités à partir de l'observation de l'objet (Savall et Zardet, 2004). Le codage de nos entretiens s'est effectué en prenant comme unité d'analyse, le dysfonctionnement. Un dysfonctionnement correspond à une anomalie ou une 
difficulté de fonctionnement d'un micro-espace ${ }^{3}$. Selon Savall et Zardet (2010), les dysfonctionnements empêchent l'entreprise de réaliser pleinement ses objectifs et d'exploiter ses ressources matérielles et humaines de manière efficiente. Nous avons ainsi considéré les anomalies ou difficultés de fonctionnement déclarés par nos interviewés dans nos entretiens comme des dysfonctionnements observés au sein de la chaîne logistique multi-acteurs de Renault Trucks.

Lors de la PHASE 2 de notre recherche-action, une deuxième réflexion a été conduite avec les acteurs de l'entreprise. L'objectif de la PHASE 2 étant d'élaborer un outil d'aide à la prise de décision stratégique afin de mieux piloter les relations intra- et inter-organisationnelles au sein de la chaîne logistique multi-acteurs de Renault Trucks. En se basant des résultats de la PHASE 1, l'attention s'est portée sur le choix et la mise en œuvre de mécanismes de coordination adéquats afin de mieux structurer les relations intra- et inter-organisationnelles et faciliter ainsi, l'intégration logistique.

Nous proposons de présenter dans la partie suivante, le cas de Renault Trucks et de détailler sa chaîne logistique multi-acteurs. Puis, dans un second temps, nous évoquons les résultats issus de la PHASE 1 de notre recherche-action afin de mettre en exergue les principaux défis à relever dans les relations intra- et inter-organisationnelles de la chaîne logistique multiacteurs. Enfin, nous proposons de présenter l'outil qui a été créé avec les acteurs de l'entreprise en s'attardant plus spécifiquement sur les critères liés à l'intégration logistique dans le pilotage des échanges intra- et inter-organisationnels au sein de la chaîne logistique multi-acteurs.

\footnotetext{
${ }^{3}$ Un micro-espace est définit par « un ensemble de personnes constituant un groupe d'activité dans un territoire géographique (service, atelier, agence) et dirigé par un même responsable hiérarchique », (Savall et Zardet, $2010:$ 194).
} 


\section{RESULTATS}

\section{La chaîne logistique multi-acteurs de Renault Trucks}

Pour comprendre le fonctionnement de cette activité internationale, nous avons réalisé une représentation de la chaîne logistique de Renault Trucks (cf. figure 2) qui a un fonctionnement de type « Order To Delivery » $\left(\mathrm{OTD}^{4}\right)$, du fournisseur au client final.

Figure 2 : La chaîne logistique multi-acteurs de Renault Trucks pour le processus OTD

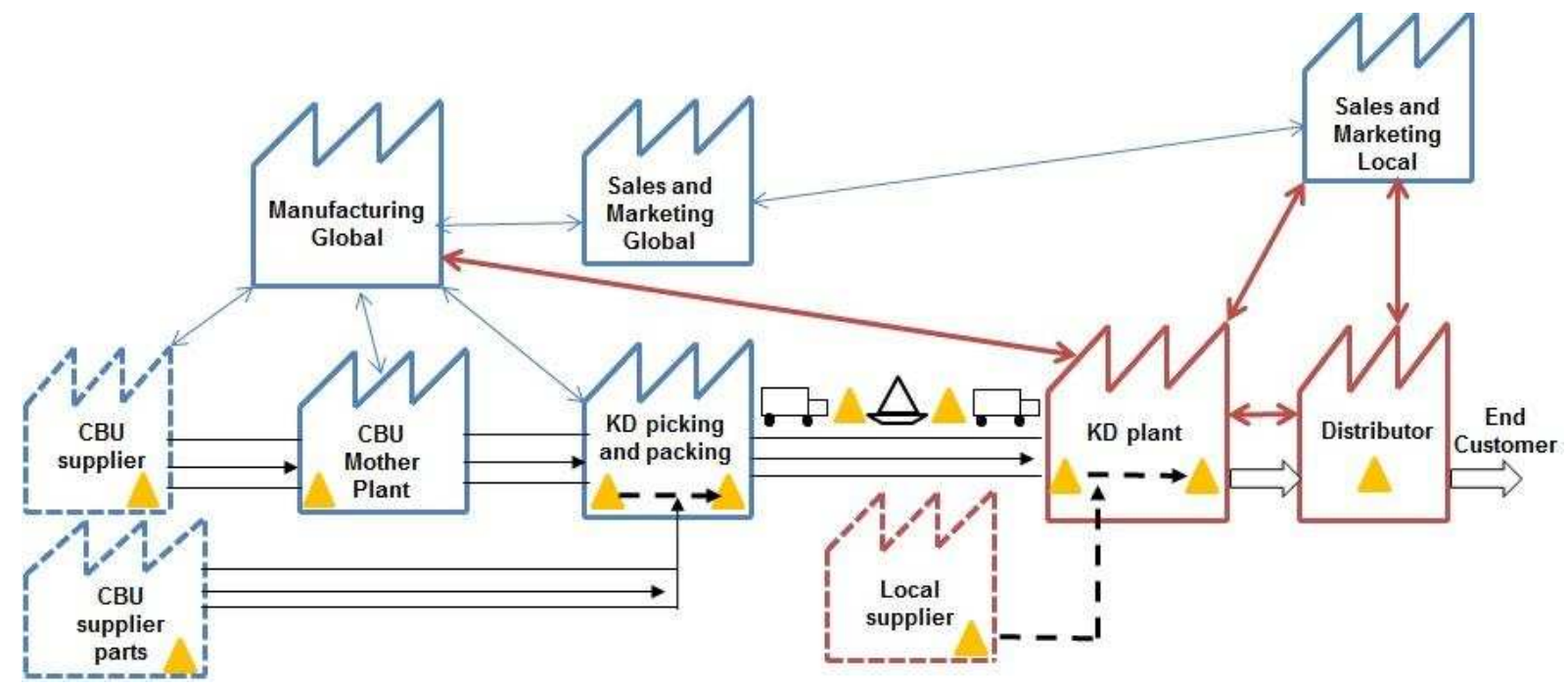

CBU: Completely Built-up Unit

KD: Knocked Down

: Stock $\leftrightarrow$ : Relations intra-organisationnelles

$\leftrightarrow$ Relations inter-organisationnelles

Source : adapté d'un document interne

La demande de clients potentiels est perçue par les départements marketing de Renault Trucks, localisés sur les marchés internationaux hors Europe (sales and marketing local). Ces départements envoient l'information au département marketing international (sales and marketing global), situé au siège social, qui centralise les demandes et formalise des commandes. Ces commandes sont envoyées au département manufacturing (manufacturing

\footnotetext{
${ }^{4}$ OTD : Order To Delivery. Pour le reste de l'article nous utiliserons cet acronyme qui symbolise le processus formalisé au sein du groupe Volvo pour traiter de la commande d'un camion jusqu'à sa livraison au client final.
} 
global) qui va les intégrer dans le système informatique de Renault Trucks. Le département Manufacturing Global ordonnance la production et la transfère aux usines d'assemblage de Renault Trucks appelées, pour les marchés européens : les usines mères (CBU mother plant). Pour la livraison des camions sur les marchés internationaux, les plateformes internationales (KD picking and packing) prélèvent directement dans l'usine mère, les pièces détachées nécessaires à l'assemblage des lots pour les marchés internationaux. Ces plateformes internationales sont situées sur le même site que les usines mères. La valeur ajoutée des plateformes internationales réside dans l'emballage des pièces détachées et la constitution de conteneurs qui seront ensuite acheminés aux différentes usines d'assemblage internationales : les assembleurs ${ }^{5}$ (KD Plant), considérés par Renault trucks comme leurs partenaires internationaux. En effet, le choix de Renault Trucks quant à sa gestion logistique internationale est d'utiliser le concept CKD (Completely Knocked Down). Le CKD désigne un mode d'approvisionnement logistique d'usines d'assemblages d'équipements. L'objectif est de créer des lots CKD contenant l'ensemble des pièces détachées nécessaires à l'assemblage d'un véhicule. Cette méthode est utilisée par les constructeurs automobiles pour assembler certains de leurs véhicules à l'étranger, dans le but de les commercialiser sur place, en utilisant des droits de douane plus faibles et de la main d'œuvre meilleur marché. Un document d'assemblage (fiches d'instructions) est livré avec les lots en CKD. Ainsi, les assembleurs assemblent les pièces détachées (en faisant parfois appel à des fournisseurs locaux) afin de produire les camions et de les apporter aux distributeurs qui auront en charge la vente et la livraison des camions aux clients finaux. Nous avons symbolisé en bleu dans la figure 2, les entités internes à Renault Trucks et en rouge les entités externes. Les flèches bleues illustrent les relations intra-organisationnelles entre les différentes entités appartenant à Renault Trucks. Les flèches rouges illustrent les relations inter-organisationnelles (soit entre

\footnotetext{
${ }^{5}$ Dans le cas de Renault Trucks, les assembleurs sont situés dans des pays tels que l'Iran, l'Irak, l'Uruguay, la Malaisie, l'Afrique du Sud et la Turquie.
} 
les entités de Renault Trucks et les assembleurs, soit entre les assembleurs et les distributeurs qui peuvent être des entreprises différentes).

\section{Les principaux défis à relever au sein de la chaîne logistique multi-acteurs}

Un recensement de l'ensemble des dysfonctionnements exprimés par les interviewés durant nos entretiens de la PHASE 1 a été conduit. Nous avons ainsi comptabilisé 68 dysfonctionnements $^{6}$. A partir de ces dysfonctionnements recensés, nous avons organisé une journée de travail avec les acteurs de Renault Trucks impliqués dans le pilotage des relations de la chaîne logistique (25 participants). Des groupes de discussion interactifs (Balogun, et al., 2003) ont été organisés afin de trouver les causes racines de ces dysfonctionnements et de déterminer les grands thèmes inhérents au pilotage des relations intra- et interorganisationnelles de la chaîne logistique multi-acteurs (cf. figure 1). Une confrontation collective des résultats avec les acteurs de l'entreprise a permis d'élaborer une représentation commune des principales causes racines des dysfonctionnements. Il en résulte trois principaux thèmes synthétisant les problématiques évoquées par les interviewés : la formulation d'une stratégie globale, l'organisation interne des processus et les échanges inter-organisationnels. En reprenant notre grille d'analyse développée dans la première partie de cet article, nous illustrons les résultats ainsi obtenus (cf. figure 3).

\footnotetext{
${ }^{6}$ Ces dysfonctionnements sont des données déclaratives et ne peuvent être interprétées comme le reflet d'une mauvaise performance du système de production de Renault Trucks. Ils reflètent simplement les améliorations possibles sur le fonctionnement interne du département.
} 
Figure 3 : Les principaux défis à relever au sein de la chaîne logistique

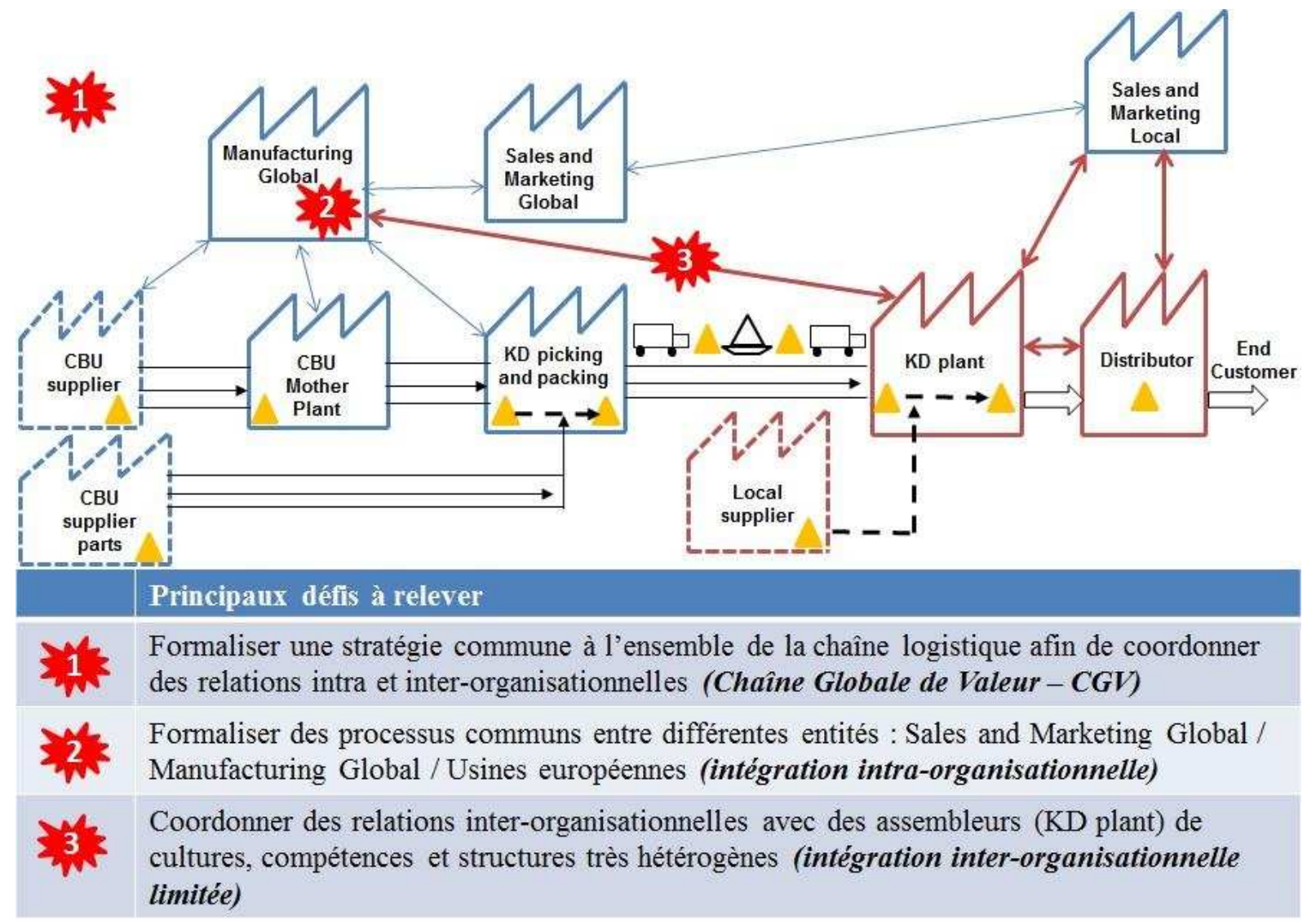

Ces trois thèmes reflètent très bien les enjeux que Renault Trucks doit relever afin d'améliorer l'intégration logistique dans le pilotage de ses relations intra- et inter-organisationnelles avec les assembleurs internationaux (KD plant). D'une part, l'analyse des causes racines de dysfonctionnements établies à un niveau macro de la chaîne logistique avec l'approche CGV, nous autorise à penser qu'une formalisation de la stratégie au niveau global de la chaîne logistique semble difficile à mettre en place mais nécessaire pour toute entreprise souhaitant intégrer des relations multiples autour d'objectifs communs, autrement dit, pour mettre en place un SCM sur l'ensemble de la chaîne logistique. D'autre part, pour piloter des relations inter-organisationnelles dans la chaîne logistique, cela présuppose auparavant, une formalisation claire des processus à un niveau intra-organisationnel. Le département manufacturing international n'est pas asez intégré dans les autres départements de la FMN, notamment dans les processus de fabrication et de commercialisation des véhicules industriels 
sur les marchés européens. Enfin, en se focalisant plus spécifiquement sur le pilotage des relations inter-organisationnelles par la FMN dans une volonté d'optimiser les flux logistiques sur l'ensemble de la chaîne logistique, une attention particulière est à porter sur la gestion des ressources et des compétences détenues par les partenaires internationaux (intégration interorganisationnelle limitée). En effet, les partenaires internationaux sont très hétérogènes et cette diversité augmente les difficultés d'intégration logistique.

A partir de ces premiers résultats, la PHASE 2 de notre recherche-action a eu pour objectif d'élaborer un outil d'aide à la prise de décision stratégique en termes de choix et mise en œuvre de mécanismes de coordination pour améliorer le pilotage des relations intra- et interorganisationnelles au sein de la chaîne logistique multi-acteurs plus particulièrement dans la relation entre Renault Trucks et ses partenaires internationaux (assembleurs), dont certains sont détenus à 100\% par Renault trucks (relation intra-organisationnelle) et d'autres sont des entreprises locales (relation inter-organisationnelle). Dans l'analyse de nos 29 entretiens issus de la PHASE 1 de notre recherche et des discussions collectives sur le pilotage globale de la chaîne logistique internationale avec les principaux acteurs concernés, le concept d'autonomie est apparu comme transversal aux différents thèmes évoqués précédemment. C'est donc autour de la notion d'autonomie que la construction de cet outil a été élaborée.

\section{L'évaluation du niveau d'autonomie des assembleurs}

L'objectif a donc été de réaliser une grille d'évaluation du niveau d'autonomie des différents assembleurs internationaux. 14 entretiens directifs ont été réalisés avec les responsables de chaque fonction de la chaîne logistique qui établissent des relations avec les assembleurs dans le cadre de leur travail: manufacturing (outillage, documentation, électronique, sourcing local, emballage, gestion des réclamations), logistique, qualité produit, qualité process, contrôle de gestion et responsables de zones géographiques (chefs de projets). Nous avons 
demandé à nos interviewés de définir des critères d'évaluation afin d'apprécier le niveau d'autonomie des différents assembleurs. Un total de 28 critères ont ainsi été proposés par les interviewés (cf. tableau 6). Pour renforcer cette grille et tester sa cohérence globale afin de la rendre la plus opérationnelle possible, nous avons réuni les différents responsables de zones géographiques et le directeur d'un des assembleurs afin de l'appliquer à trois cas d'assembleurs (Afrique du Sud, Turquie et Uruguay) faisant partie de la chaîne logistique de Renault Trucks. Suite à cela, certains critères ont été modifiés, supprimés, clarifiés et rajoutés.

Tableau 6 : La grille d'évaluation du niveau d'autonomie des assembleurs

\begin{tabular}{|c|c|c|c|c|c|}
\hline & \begin{tabular}{|} 
Nbre \\
de \\
critères
\end{tabular} & $\begin{array}{l}\text { Critères liés à l'assembleur } \\
\text { en termes de : }\end{array}$ & A & SA & NA \\
\hline $\begin{array}{l}\text { Manufacturing } \\
\text { engineering }\end{array}$ & 11 & $\begin{array}{l}\text { documentation, outillage, électronique, } \\
\text { sourcing local, emballage }\end{array}$ & & & \\
\hline Logistique & 4 & $\begin{array}{l}\text { fonction logistique en local chez } \\
\text { l'assembleur, connaissances et pratiques des } \\
\text { concepts }\end{array}$ & & & \\
\hline Qualité & 4 & $\begin{array}{c}\text { Traitement des non-conformités, réalisation } \\
\text { d'audits interne }\end{array}$ & & & \\
\hline Système de production & 3 & $\begin{array}{l}\text { Polyvalence des compétences, amélioration } \\
\text { continue, plan de surveillance }\end{array}$ & & & \\
\hline Contrôle de gestion & 1 & Gestion interne des coûts & & & \\
\hline Facteurs contextuels & 5 & $\begin{array}{l}\text { Moyens de communication chez } \\
\text { l'assembleur, culture de travail dans } \\
\text { l'automobile, maitrise de l'anglais }\end{array}$ & & & \\
\hline
\end{tabular}

Nous présentons plus spécifiquement les critères établis avec les acteurs de l'entreprise sur la partie logistique des relations avec les assembleurs internationaux. Il en résulte quatre critères. Dans notre volonté d'évaluer le niveau d'autonomie des assembleurs, il a été demandé aux interviewés de préciser factuellement des situations dans lesquelles il est possible d'établir objectivement le niveau d'autonomie (non-autonome, semi-autonome, autonome) de l'assembleur afin d'enlever tout aspect subjectif qui pourrait s'immiscer quant à la perception 
de nos interviewés de leurs partenaires internationaux compte tenu des relations parfois étroites qu'ils peuvent entretenir avec certains des assembleurs étudiés. Nous présentons les critères retenus pour la partie logistique (cf. tableau 7).

Tableau 7: Les critères d'évaluation du niveau d'autonomie des partenaires sur le thème logistique.

\begin{tabular}{|c|c|c|c|}
\hline Logistique & AUTONOME & SEMI-AUTONOME & NON-AUTONOME \\
\hline $\begin{array}{l}\text { Fonction logistique identifiée } \\
\text { chez l'assembleur qui possède } \\
\text { des compétences métier en } \\
\text { logistique (gestion des stocks / } \\
\text { - } 5 \text { S / planification de la } \\
\text { production / flux / } \\
\text { conditionnement ....) qui } \\
\text { permettent d'exécuter le } \\
\text { contrat de l'assembleur }\end{array}$ & $\begin{array}{c}\text { Une personne est } \\
\text { identifiée chez } \\
\text { l'assembleur ET a des } \\
\text { compétences en } \\
\text { logistique }\end{array}$ & $\begin{array}{l}\text { Une personne est } \\
\text { identifiée mais ne } \\
\text { possède pas oU peu de } \\
\text { compétences en } \\
\text { logistique }\end{array}$ & $\begin{array}{l}\text { Aucune personne chez } \\
\text { l'assembleur n'est } \\
\text { identifiée pour gérer la } \\
\text { logistique en interne }\end{array}$ \\
\hline $\begin{array}{l}\text { Respect des règles de mise en } \\
\text { œuvre des flux internes } \\
\text { logistiques (déballage / re } \\
\text { conditionnement / stockage / } \\
\text { livraison au poste de travail) }\end{array}$ & $\begin{array}{l}\text { Les règles de stockage / } \\
\text { livraison au poste de } \\
\text { travail ET de déballage / } \\
\text { re conditionnement sont } \\
\text { respectées }\end{array}$ & $\begin{array}{c}\text { Seules les règles de } \\
\text { stockage / livraison au } \\
\text { poste de travail oU les } \\
\text { règles de déballage / re } \\
\text { conditionnement sont } \\
\text { respectées }\end{array}$ & $\begin{array}{c}\text { Aucune règle en termes } \\
\text { de stockage / livraison } \\
\text { au poste de travail et de } \\
\text { déballage / re } \\
\text { conditionnement n'est } \\
\text { respectée }\end{array}$ \\
\hline $\begin{array}{l}\text { Délimitation des zones de } \\
\text { travail logistique et affectation } \\
\text { des bonnes pièces au bon } \\
\text { poste }\end{array}$ & $\begin{array}{l}\text { Les zones de travail } \\
\text { logistique sont } \\
\text { identifiées ET la gestion } \\
\text { (identification ...) des } \\
\text { pièces à chaque poste } \\
\text { est bonne }\end{array}$ & $\begin{array}{l}\text { Les zones de travail } \\
\text { logistique sont } \\
\text { identifiées OU la gestion } \\
\text { (identification ...) des } \\
\text { pièces à chaque poste } \\
\text { est bonne }\end{array}$ & $\begin{array}{c}\text { Les zones de travail ne } \\
\text { sont pas identifiées, la } \\
\text { gestion (identification ...) } \\
\text { des pièces à chaque } \\
\text { postes n'est pas effective } \\
\text { selon les standards }\end{array}$ \\
\hline $\begin{array}{l}\text { Capacité à atteindre les } \\
\text { objectifs de lead time et de } \\
\text { stock du contrat }\end{array}$ & $\begin{array}{c}\text { Processus défini } \underline{\text { AVEC }} \\
\text { des indicateurs de } \\
\text { pilotage fiables }\end{array}$ & $\begin{array}{l}\text { Processus défini SANS } \\
\text { indicateurs de pilotage } \\
\text { régulièrement actualisés }\end{array}$ & Processus non défini \\
\hline
\end{tabular}

L'outil permet aux acteurs de l'entreprise de pouvoir réaliser un diagnostic du niveau d'autonomie de l'ensemble des différents partenaires avec lesquels l'entreprise travaille. Par la suite, il sera alors possible à Renault Trucks de faire des choix quant au mode de pilotage à adopter en fonction du niveau d'autonomie de ses partenaires dans l'assemblage et la distribution de ses produits à l'échelle internationale. Ce travail de recherche a pu mettre en avant le rôle stratégique dans la nécessité de piloter les relations intra et interorganisationnelles au sein d'une chaîne logistique multi-acteurs afin de faciliter une 
intégration logistique. L'objectif de Renault Trucks est à présent tourné sur une volonté de développer l'autonomie de ses partenaires afin de favoriser une relation partenariale sur le long terme.

\section{DISCUSSION}

Une analyse de 38 articles issus des neuf revues en logistique les mieux classées proposé par Fabbe-Costes et Jahre (2008), met en évidence que les dimensions utilisées par la communauté pour définir l'intégration des chaînes logistiques sont diverses et variées. Il n'existe donc pas de consensus sur les outils de mesure de la notion d'intégration logistique et plus particulièrement sur le lien qui en est fait avec leur performance. Il est ainsi préconisé par certains chercheurs de mobiliser plusieurs niveaux d'intégration afin de contribuer à la performance d'une chaîne logistique (Sahin et Robinson, 2005), notamment de prendre en compte une intégration intra et inter-organisationnelle (Rodrigues, et al., 2004). Ainsi, nos résultats confirment l'utilisation d'une approche multi-niveau de la notion d'intégration au sein des chaînes logistiques multi-acteurs afin de positionner les principaux défis à relever dans le pilotage des relations intra- et inter-organisationnelles au sein du système de production international d'une FMN. En effet, l'analyse dysfonctionnelle de 29 entretiens semi-directifs auprès d'acteurs impliqués dans la chaîne logistique de Renault Trucks et une journée de travail avec les responsables des départements marketing, manufacturing et les responsables des fonctions (qualité, méthodes, logistiques, plateformes internationales, outillages, contrôle de gestion, emballages ; 25 participants), mettent en avant les principaux défis à relever dans le pilotage des relations au sein d'une chaîne logistique multi-acteurs. Nous avons ainsi positionné nos résultats en utilisant une grille d'analyse multi-niveau : CGV, intégration intra-organisationnelle et intégration inter-organisationnelle limitée. Ce triptyque [CGV - intégration intra-organisationnelle - intégration inter-organisationnelle limitée] a permis d'appréhender les problématiques liées au pilotage des relations partenariales dans 
notre recherche-action avec Renault Trucks. Positionner ce triptyque dans une vision systémique permet d'identifier les principaux thèmes qui révèlent l'enjeu stratégique pour une FMN à piloter sa chaîne logistique multi-acteurs afin de favoriser l'intégration logistique sur l'ensemble de celle-ci.

Par ailleurs, cette approche différenciée de l'intégration logistique selon plusieurs niveaux peut aider les entreprises à identifier et se focaliser sur des éléments clés d'intégration (FabbeCostes, 2008). C'est ce que nous avons observé dans notre recherche-action avec Renault Trucks qui, à la suite de la représentation collective des principaux défis à relever dans sa chaîne logistique multi-acteurs, a décidé de focaliser son attention sur un élément clé d'intégration à savoir, les mécanismes de coordination dans le pilotage des relations intra- et inter-organisationnelles. Des mécanismes de coordination appropriés entre Renault trucks et ses partenaires internationaux sont alors considérés comme des facteurs facilitateurs pour une intégration logistique multi-niveau. A l'issue de notre recherche-action, nous émettons la proposition que le degré d'autonomie/d'hétéronomie du partenaire peut-être une variable influençant les choix stratégiques de la FMN en termes de contrôle et de coordination des échanges dans l'objectif d'une intégration logistique au sein de son système de production. En effet, plus un partenaire international est autonome sur les différents critères établis dans l'outil conçu avec les acteurs de Renault Trucks, plus l'intégration de la logistique au sein de la chaîne logistique sera alors conduite avec facilité. Comme nous avons pu l'observer au travers du cas de Renault Trucks, la chaîne logistique intègre de plus en plus d'acteurs et d'entités hétérogènes qui intensifient les interactions à un double niveau (intra- et interorganisationnel) qu'il convient de piloter efficacement. Sur ce point, l'étude de Cousins et Menguc (2006) montre que l'intégration des acteurs au sein de la chaîne logistique a un impact sur la performance de celle-ci. Ainsi, l'une des principales difficultés réside dans le fait de pouvoir piloter ces interactions et d'instaurer une réflexion afin d'identifier et 
formaliser des processus pour mettre en avant une gestion coordonnée et flexible des relations intra- et inter-organisationnelles au sein de l'ensemble de la chaîne logistique. C'est d'ailleurs en ce sens que certains auteurs évoquent la nécessité de mettre en place des mécanismes de contrôle pour favoriser le maintien de relations partenariales sur le long terme (Brulhart et Favoreu, 2006) où la confiance joue un rôle prépondérant dans les échanges relationnels (Carbone, 2005). Ces problématiques d'intégration des acteurs plus particulièrement de coordination, sont très bien mis en avant par la théorie de l'entreprise-réseau comme évoqué par Baudry (2004) dans sa définition de la firme-réseau: «Une firme-réseau regroupe contractuellement un ensemble de firmes juridiquement indépendantes reliées verticalement au sein duquel une firme-pivot coordonne de manière récurrente des opérations d'approvisionnement, de production et de distribution »(Baudry, 2004: 250). L'impératif de coordonner les activités des firmes membres du réseau relevant de la sphère de la production devient alors crucial. C'est ainsi que le rôle de la firme pivot (dans notre cas Renault Trucks) est stratégiquement important dans l'intégration logistique intra- et inter-organisationnelle au sein d'une chaîne logistique multi-acteurs. Cependant, la notion d'autonomie est peu traitée dans cette littérature particulièrement sur le fait que le niveau d'autonomie/hétéronomie permettrait à la firme-pivot de choisir et mettre en place des mécanismes de coordination spécifiques afin de faciliter l'intégration des acteurs tout au long de sa chaîne logistique multiacteurs. Un certain nombre de travaux s'attachent à expliquer la configuration des entreprises en réseau, mais peu d'entre eux, se consacrent à leur fonctionnement effectif (Roveillo, et al., 2012). Ainsi, nous préconisons une nouvelle variable qu'il convient, selon nous, d'étudier de façon plus approfondie à l'avenir. En outre, nous proposons d'identifier le niveau d'autonomie/hétéronomie du partenaire comme variable permettant à la firme-pivot de coordonner plus efficacement des relations intra- et inter-organisationnelles au sein de sa 
chaîne logistique multi-acteurs constituant ainsi un facteur facilitateur d'une intégration logistique de l'entreprise-réseau.

\section{CONCLUSION}

La fragmentation de la chaîne de valeur s'accompagne d'une dispersion des activités des firmes multinationales et a des conséquences sur la gestion des interfaces entre les différents acteurs/sites au sein de l'organisation étendue. Notre travail s'est focalisé sur la notion d'intégration logistique au sein d'une chaîne multi-acteurs de Renault Trucks dans la fabrication et la commercialisation de ses camions sur les marchés internationaux hors Europe. A l'aide d'une analyse dysfonctionnelle de 29 entretiens semi-directifs et l'élaboration d'un outil d'aide au diagnostic et à l'action stratégique avec les acteurs de l'entreprise, nous avons pu présenter deux contributions à ce travail de recherche. D'une part, analyser la notion d'intégration logistique d'une chaîne multi-acteurs, nécessite une analyse multi-niveaux. Nous proposons le triptyque suivant:[approche CGV - intégration intraorganisationnelle - intégration inter-organisationnelle]. D'autre part, nous avons pu montrer que l'intégration logistique d'une chaîne multi-acteurs est facilitée par la mise en place de mécanismes de coordination spécifiques. Le choix de ces mécanismes de coordination dépend du niveau d'autonomie/hétéronomie du partenaire international.

\section{REFERENCES}

Argyris, C. (1995), Savoir pour agir. Surmonter les obstacles à l'apprentissage organisationnel, InterÉditions, Paris.

Bair, J. (2010), Les cadres d'analyse des chaînes globales, Revue Française de Gestion, $\mathrm{N}^{\circ}$ 201, pp. 103-119.

Balogun, J., Sigismund Huff, A. , Jhonson, P. (2003), Three Responses to the Methodological Challenges of Studying Strategizing, Journal of Management Studies, Vol. 40, $\mathrm{N}^{\circ}$ 1, pp. 197-224.

Bartlett, C.A., Beamish, P.W. (2011), Transnational Management: Text, Readings, and Cases in Cross-Border Management, 6th édition, Burr Ridge, IL: Irwin McGraw-Hill. 
Baudry, B. (2004), La question des frontières de la firme. Incitation et coordination dans la firme-réseau, Revue économique, Vol. 55, $\mathrm{N}^{\circ}$ 2, pp. 247-273.

Buckley, P.J, Ghauri, P.N. (2004), Globalisation, Economic geographie and the Strategy of multinational enterprises, Journal of International Business Studies, Vol.35, N², pp.81-98.

Brulhart, F., Favoreu, C. (2006), Le lien contrôle-confiance-performance dans les relations de partenariat logistique inter firmes, Finance Contrôle Stratégie, Vol. 9, № 5, pp. 59-96.

Carbone, V. (2005), L'ampleur de la relation client-fournisseur: le cas des alliances logistiques, Logistique \& Management, Vol. 13, $\mathrm{N}^{\circ}$ 1, pp.89-98.

Chanal, V., Lesca H., Martinet A.-C. (1997), Vers une ingénierie de la recherche en sciences de gestion, Revue Française de Gestion, N 116, pp. 41-51.

Colin, J. (2005), Le supply chain management existe-t-il réellement ?, Revue Française de Gestion, N¹56, pp. 135-149.

Colovic, A., Mayrhofer, U. (2011), Optimizing the Location of R\&D and Production Activities: Trends in the Automotive Industry, European Planning Studies, Vol.19, $\mathrm{N}^{\circ} 8$, pp.1481-1498.

Cousins, P.D., Menguc, B. (2006), The implications of socialization and integration in supply chain management, Journal of Operations Management, Vol. 24, $\mathrm{N}^{\circ}$ 5, pp. 604-620.

David, A. (2008), Logique, épistémologie et méthodologie en sciences de gestion : trois hypothèses revisitées, in A. David, A. Hatchuel, R. Laufer ( $2^{\text {ème }}$ éd.), les nouvelles fondations des sciences de gestion. Eléments épistémologiques de la recherche en management, VuibertFnege, Paris.

Fabbe-Costes, N. (2007), La gestion des chaînes logistiques multi-acteurs: les dimensions organisationnelles d'une gestion lean et agile, in G. Paché, A. Spalanzani (coord.), La Gestion des chaînes logistiques multi-acteurs: perspectives stratégiques, Presses Universitaires de Grenoble, Grenoble.

Fabbe-Costes, N., Jahre, M. (2008), Supply chain integration and performance: a review of the evidence, The International Journal of Logistics Management, Vol. 19, № 2, pp. 130-154.

Fabbe-Costes, N., Lancini, A. (2009), Gestion inter-organisationnelle des connaissances et gestion des chaînes logistiques : enjeux, limites et défis, Management \& Avenir, $\mathrm{N}^{\circ}$ 24, pp. 123-145.

Fulconis, F., Paché G., Roveillo, G. (2011), La prestation logistique. Origines, enjeux et perspectives, éditions EMS, Paris.

Gereffi, G. (1994), The organization of buyer-driven global commodity chains: how US retailers shape overseas production networks, in G. Gereffi and M. Korzeniewicz (eds) Commodity Chains and Global Capitalism, Westport, CT, Praeger. 
Gereffi, G., Humphrey, J., Sturgeon, T. (2005), The governance of global value chains, Review of International Political Economy, Vol. 12, Nº1, pp.78-104.

Livolsi, L. (2007), Contrôle de gestion et pilotage stratégique des chaînes logistiques multiacteurs, in G. Paché, A. Spalanzani (coord.), La Gestion des chaînes logistiques multiacteurs : perspectives stratégiques, Presses Universitaires de Grenoble, Grenoble.

Livolsi, L. (2009), Le Supply Chain Management : synthèse et propositions, Actes de la XVIIIème Conférence Internationale de Management Stratégique de l'AIMS, 2 au 5 juin 2009, Grenoble.

Mayrhofer, U. (2011), La gestion des relations siège-filiales, Revue Française de Gestion, Vol.3, $\mathrm{N}^{\circ} 212$, pp.65 à 75.

Mayrhofer, U., Urban, S. (2011), Management International : des pratiques en mutation, Pearson Education France, Paris.

Paché, G., Spalanzani, A. (2007), La Gestion des chaînes logistiques multi-acteurs : perspectives stratégiques, Press Universitaire de Grenoble.

Porter, M.E. (1985), Competitive advantage, Creating and Sustaining Superior Performance, The Free Press, New York.

Rodrigues, A.M., Stank, T.P., Lynch D.F. (2004), Linking strategy, structure, process, and performance in integrated logistics, Journal of Business Logistics, Vol. 25, N 2, pp. 65-94.

Roveillo, G., Fulconis, F., Paché, G. (2012), Vers une dilution des frontières de l'organisation: le prestataire de services logistiques (PSL) comme pilote aux interfaces, Logistique \& Management, Vol. 20, $\mathrm{N}^{\circ}$ 2, pp. 7-20.

Sahin, F., Robinson E.P. Jr (2005), Information sharing and coordination in make-to-order supply chains, Journal of Operations Management, Vol. 23, № 6, pp. 579-598.

Savall, H., Zardet, V. (1997), Vers la pensée en action stratégique ou le non-dit dans le discours sur la stratégie - Propositions pour améliorer la qualité scientifique des recherches en stratégie, Management International, Vol. 2, $\mathrm{N}^{\circ}$ 1, pp. 77-96.

Savall, H., Zardet V. (2004), Recherche en Sciences de Gestion : approche qualimétrique. Observer l'objet complexe, Economica, Paris.

Savall, H. et Zardet, V. (2010), Maîtriser les coûts et les performances cachés, Economica, Paris.

Sturgeon, T. (2009), From commodity chains to value chains: interdisciplinary theory building in an age of globalization, Frontiers in commodity chains research, Bair J. (Ed.), Stanford University Press, pp. 110-135.

Yin, R.K. (2003), Case study research: design and methods, 3e éd., Thousand Oaks : Sage. 